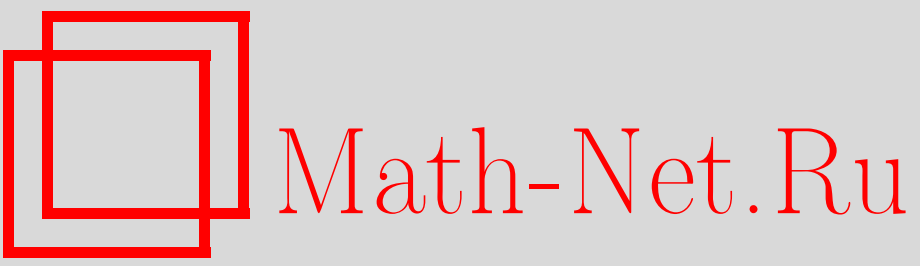

А. А. Туганбаев, Модули над наследственными и полуцепными кольцами, УМH, 1999, том 54, выпуск 5, 177-178

DOI: https://doi.org/10.4213/rm221

Использование Общероссийского математического портала Math-Net.Ru подразумевает, что вы прочитали и согласны с пользовательским соглашением

http://www.mathnet.ru/rus/agreement

Параметры загрузки:

IP : 52.23 .180 .231

26 апреля 2023 г., 16:17:04 


\title{
МОДУЛИ НАД НАСЛЕДСТВЕННЫМИ И ПОЛУЦЕПНЫМИ КОЛЬЦАМИ
}

\author{
А. А. ТуГАНБаЕв
}

Все колца предполагаются ассоциативными и с ненулевой единицей. Слова типа "нётерово кольцо" означают, что соответствующие условия выполнены справа и слева. Модуль $M$ назьвается $\pi$-проективнылм, если каждый идемпотентный эндоморфизм любого фактор-модуля модуля $M$ поднимается до эндоморфизма модуля $M$. Модуль $M$ назьвается $\pi$-инвективнылм, если каждый идемпотентный эндоморфизм любого подмодуля модуля $M$ продолжается до эндоморфизма модуля $M$. Модуль $M$ называется квазипроективным, если для любого эпиморфизма $h: M \rightarrow \bar{M}$ и каждого гомоморфизма $\bar{f}: M \rightarrow \bar{M}$ существует такой гомоморфизм $f: M \rightarrow M$, что $h f=\bar{f}$. Каждый квазипроективный (квазиинъективньй) модуль является $\pi$-проективншм ( $\pi$-инъективным) модулем. Модуль называется цепнылм, если любые его два подмодуля сравнимы по включению. Прямая сумма цепных модулей назьвается полуцепньим модулем. Модуль называется наследственныцм, если все его подмодули проективны. Классическое правое кольцо частных кольца $A$ обозначается через $Q_{c l}(A)$. Цепная нётерова область $A$ называется полной, если кольцо $A$ является полным относительно $J(A)$-адической топологии.

ЗАмечание 1 . Пусть $A$ - наследственное нётерово первичное кольцо, не являющееся примитивным справа кольом. В теореме 8 из [1] выяснено, когда $Q_{c l}(A)_{A}$-квазипроективный модуль.

Замечание 2 [2]. Пусть $A$ - наследственное нётерово первичное кольцо. Тогда

$Q_{c l}(A)_{A}-\pi$-проективный модуль $\Longleftrightarrow$

либо $A$ - цепная нётерова область, либо существует такое натуральное число $n$, что колцо $A$ изоморфно кольцу всех $(n \times n)$-матриц над некоторой полной цепной нётеровой областью $D$.

Тогда

ЗАмечание 3 [2]. Пусть $A$ - наследственное справа нётерово справа полупервичное кольцо.

$Q_{c l}(A)_{A}$ - прямая сумма $\pi$-проективных модулей $\Longleftrightarrow$

$A$ - полуцепное кольцо.

ЗАмЕЧАНИЕ 4 [3; теорема 11.75].

$A$ - полуцепное слева нётерово справа полупервичное кольцо $\Longleftrightarrow$

$A$ - полуцепное справа нётерово слева полупервичное кольцо $\Longleftrightarrow$

$A$ - конечное прямое произведение полуцепных нётеровых наследственных первичных колец.

Теорема 1. Пусть А-наследственное справа нётерово справа полупервичное кольио. Тогда:

(1) $Q_{c l}(A)_{A}-$ прямая сумма $\pi$-проективных модулей $\Longleftrightarrow$

$A$ - конечное прямое произведение полуцепных нётеровых наследственных первичнвьх колец.

(2) $Q_{c l}(A)_{A}-\pi$-проективный правый $A$-модуль $\Longleftrightarrow$

$A=A_{1} \times \cdots \times A_{n}$, где каждое кольцо $A_{i}$ либо является чепной нётеровой областью, либо изоморфно кольцу всех $(n(i) \times n(i))$-матриц над некоторой полной чепной нётеровой областью $D_{i}($ әде $n(i)$ - натуральнье числа).

ДокАЗАТЕЛЬСтво. (1) следует из замечаний 3 и 4.

(2) В силу (1) можно без ограничения общности считать, что $A$ - наследственное нётерово первичное кольцо. В этом случае утверждение следует из замечания 2.

ЗАмечАниЕ 5. Существует цепная нётерова область $A$, не являющаяся ни примитивной справа, ни полной (например, в качестве $A$ можно взять кольцо всех рациональных чисел с нечетными знаменателями). Поэтому из теоремы 1 следует, что существует такое наследственное нётерово первичное кольцо $A$, что $A$ не является примитивным справа кольцом, и $Q_{c l}(A)_{A}-\pi$-проективный

Работа выполнена при поддержке Российского фонда фундаментальных исследований (грант № 99-01-00382). 
модуль, не являющийся квазипроективным. Следователњно, даже в непримитивном случае, существуют кольца, подпадающие под теорему 1 и не подпадающие под вышеупомянутую теорему 8 из [1].

ЗАмечание 6 [3; предложение 4.13]. Пусть $E$ - инъективная оболочка модуля $M$. Тогда

$M-\pi$-инъективный модуль $\Longleftrightarrow$

$M=\bigoplus_{i \in I}\left(E_{i} \bigcap M\right)$ для любого прямого разложения $E=\bigoplus_{i \in I} E_{i}$.

Модуль называется равномерным, если любые два его ненулевых подмодуля имеют ненулевое пересечение. Прямая сумма равномерных модулей называется полуравномернымм модулем. Правый модуль $M$ над кольцом $A$ называется несингулярныцм, если аннулятор в кольц $A$ любого ненулевого элемента модуля $M$ не является существенным правым идеалом кольца $A$.

ЗАмечание $7 . A$ - нётерово справа полуцепное кольцо $\Longleftrightarrow$

$A$ - полуравномерное справа кольцо и все инъективные правые $A$-модули являются полуцепными $\Longleftrightarrow$

$A$ - полуравномерное справа кольцо и все $\pi$-инъективные правые $A$-модули являются полуцепными.

(Первая эквивалентность из замечания 7 доказана в [4; 3.61]. Вторая эквивалентность следует из замечания 6.)

ЗАмечАниЕ 8 [5]. Каждый конечнопорожденный несингулярный модуль над полуцепным кольцом является полуцепным проективным модулем.

ЗАмечАниЕ 9 [6]. Каждый инъективный несингулярный модуль над полуцепным кольцом является полуцепным модулем.

ТЕОРема 2. Пусть $M-\pi$-инбективный несингулярный правый модуль над полуцепньцм кольцом $A$.

Тогда $M$ - полуцепной модуль, причем $M=N \oplus P$, где $N$ - инвективный полуцепной модуль, $P$ - полуцепной модуль, являющийся подмодулем некоторого проективного A-модуля.

Если, кроме того, А - нётерово справа кольцо, то $P$ - проективный модуль.

ДокАЗАтЕльСтво. Инъективная оболочка $E$ модуля $M$ является несингулярным модулем. По замечанию $9 E=\bigoplus_{i \in I} E_{i}$, где все $E_{i}$ - цепные модули. По замечанию $6 M$-прямая сумма цепных модулей $M_{i}=E_{i} \cap M$. Пусть $J$ - подмножество множества индексов $I$, образованное всеми такими индексами $i$, что $M_{i}=E_{i}, L \equiv I \backslash J, N \equiv \bigoplus_{i \in J} M_{i}, P \equiv \bigoplus_{i \in L} M_{i}, F \equiv$ $\bigoplus_{i \in L} E_{i}$, причем если $J(L)$ - пустое множество, то полагаем $N=0(P=F=0)$. Тогда $E \stackrel{=}{=} \oplus F, M=N \oplus P, N$ - инъективный полуцепной модуль. По определению модуля $F$ для любого $i \in L$ существуют элементы $q_{i} \in E_{i} \backslash M_{i}$. Пусть $Q \equiv \bigoplus_{i \in L} q_{i} A$. По замечанию 8 все циклические несингулярные модули $q_{i} A$ являются проективными модулями. Поэтому $Q-$ проективный модуль. Так как все $E_{i}$ - цепные модули, то $M_{i} \subseteq q_{i} A$ для любого $i \in L$. Поэтому $P \subseteq Q$. Допустим теперь, что $A$ нётерово справа. Тогда при $i \in L$ все циклические модули $q_{i} A$ являются нётеровыми модулями. Поэтому при $i \in L$ все модули $M_{i}$ конечно порождены. По замечанию 8 при $i \in L$ все конечнопорожденные несингулярные модули $M_{i}$ проективны. Поэтому $P$ - проективный модуль.

\section{СПИСОК ЛИТЕРАТУРЫ}

[1] Singh S. // Canad. J. Math. 1974. V. 26. № 5. P. 1173-1185. [2] Туганбаев A. А. // Матем. сб. 1998. T. 189. № 4. C. 143-160. [3] Tuganbaev A. A. Semidistributive Modules and Rings. Dordrecht: Kluwer, 1998. [4] Tuganbaev A. A. Distributive Modules and Related Topics. Amsterdam: Gordon and Breach, 1999. [5] Warfield R. B. // J. Algebra. 1975. V. 37. № 3. P. 187-222. [6] Туганбаев А. А. // Матем. заметки. 1999. Т. 65. №6. С. 880-892.

Московский энергетический институт

(технический университет)

E-mail: askar@tuganbaev.mccme.ru
Принято редколлегией 23.08.1999 